Series A

I. MATHEMATICA

307

\title{
ON PERIODIC INFINITE RADICALS
}

BY

GEORGELLEN SCHUSKE and W. J. THRON

University of Colorado

Boulder, Colorado

H E L S I N K I 1962

S U O M A L A I N E N T I E D E A K A T E M I A

https://doi.org/10.5186/aasfm.1962.307 
Communicated 8 September 1961 by P. J. Myrberg and K. VÄIsäL̈̈ 


\section{On periodic infinite radicals}

If $\left\{a_{n}\right\}$ is a sequence of complex numbers, the sequence $\left\{u_{n}\right\}$, where

$$
u_{n}=\sqrt{a_{1}+\sqrt{a_{2} \ldots+\sqrt{a_{n}}}},
$$

is called an infinite radical. The numbers $a_{j}$ are called the elements of the

infinite radical. Throughout this paper $\sqrt{a}$ will mean that square root whose real part is positive or zero. If $a$ is a negative real number, $\sqrt{a}$ will be defined to lie on the positive imaginary axis.

The convergence of infinite radicals with complex elements has been investigated by the authors [3]. Unbounded conditional convergence regions were developed by using the Stieltjes-Vitali Theorem for normal families of functions in conjunction with a criterion given by Herschfeld [1] for infinite radicals with positive real elements. A short historical survey and a list of references to the literature on infinite radicals were included. Recently, Myrberg [2] has considered infinite radicals from a different point of view.

The present paper deals with infinite radicals in which all elements are equal, that is, periodic infinite radicals of period one. Although many of the results can be deduced from our earlier paper, the elementary proofs given here are to be preferred for this special case because in them no function theory is used. Moreover, the case of negative real elements, which was not considered in the earlier study, is included here.

Theorem: Let a be any complex number. Then the infinite radical $\left\{u_{n}\right\}$, where

$$
\begin{aligned}
u_{1} & =\sqrt{a}, \\
u_{n+1} & =\sqrt{a+u_{n}}, \quad n=1,2, \ldots,
\end{aligned}
$$

converges, and

$$
\lim _{n \rightarrow \infty} u_{n}=\frac{1+\sqrt{1+4 a}}{2},
$$

where $\sqrt{1+4 a}$ is taken in accordance with the above definition.

This research was supported by the United States Air Force under contract no. AF 49(638)-100 monitored by the AF Office of Scientific Research of the Air Research and Development Command. 
Proof: If $\lim u_{n}$ exists, it must be a root of the equation $n \rightarrow \infty$

$$
z^{2}-z-a=0
$$

since

$$
\lim _{n \rightarrow \infty} u_{n+1}=\lim _{n \rightarrow \infty} \sqrt{a+u_{n}}
$$

Let $u$ and $u^{*}$ be the roots of this equation:

$$
u=\frac{1+\sqrt{1+4 a}}{2}, \quad u^{*}=\frac{1-\sqrt{1+4 a}}{2} .
$$

For every $n$, write

$$
u_{n}=\alpha_{n}+i \beta_{n}
$$

and

$$
\gamma=\mathrm{R}\left(\frac{\sqrt{1+4 a}}{2}\right), \quad \eta=\mathrm{I}\left(\frac{\sqrt{1+4 a}}{2}\right)
$$

Since the proof for the case $a=-\frac{1}{4}$ is basically different from that for other values of $a$, we shall consider this exceptional case last. For $a \neq-\frac{1}{4}$, define

$$
R_{n}=\frac{u_{n}-u}{u_{n}-u^{*}}
$$

We shall prove that $\lim _{n \rightarrow \infty} R_{n}=0$, and it will then follow that $\lim _{n \rightarrow \infty} u_{n}=u$.

From the equations

$$
u_{n+1}^{2}=u_{n}+a, \quad u^{2}=u+a, \quad u^{* 2}=u^{*}+a,
$$

we have

$$
u_{n+1}^{2}-u^{2}=u_{n}-u \quad \text { and } \quad u_{n+1}^{2}-u^{* 2}=u_{n}-u^{*} .
$$

Then

$$
\begin{aligned}
\frac{R_{n+1}}{R_{n}} & =\frac{u_{n+1}-u}{u_{n+1}-u^{*}} \cdot \frac{u_{n}-u^{*}}{u_{n}-u} \\
& =\frac{u_{n+1}-u}{u_{n+1}-u^{*}} \cdot \frac{u_{n+1}^{2}-u^{* 2}}{u_{n+1}^{2}-u^{2}} \\
& =\frac{u_{n+1}+u^{*}}{u_{n+1}+u} .
\end{aligned}
$$


Case 1(a): a nonreal. If I $(a)>0$, it is clear that $\gamma, \eta>0$. Also, for every $n, \alpha_{n}>0$ because all square roots will lie in the first quadrant, and $\beta_{n}>0$ since a finite number of root extractions of nonreal quantities cannot yield a real number. Then

$$
\begin{aligned}
\left|\frac{R_{n+1}}{R_{n}}\right| & =\left|\frac{\alpha_{n+1}+i \beta_{n+1}+\frac{1}{2}-\gamma-i \eta}{\alpha_{n+1}+i \beta_{n+1}+\frac{1}{2}+\gamma+i \eta}\right| \\
& =\sqrt{\frac{\left(\alpha_{n+1}+\frac{1}{2}-\gamma\right)^{2}+\left(\beta_{n+1}-\eta\right)^{2}}{\left(\alpha_{n+1}+\frac{1}{2}+\gamma\right)^{2}+\left(\beta_{n+1}+\eta\right)^{2}}}
\end{aligned}
$$

Thus

$$
\begin{aligned}
\left|\frac{R_{n+1}}{R_{n}}\right| & =\sqrt{1-\frac{4 \gamma \alpha_{n+1}+2 \gamma+4 \eta \beta_{n+1}}{\left|u_{n+1}+u\right|^{2}}} \\
& <\sqrt{1-\frac{2 \gamma}{\left|u_{n+1}+u\right|^{2}}} .
\end{aligned}
$$

By repeated use of the triangle inequality,

$$
\begin{aligned}
\left|u_{n}\right| & <\sqrt{|a|+\sqrt{|a|+\ldots+\sqrt{|a|}}} \quad(n \text { elements }) \\
& <\frac{1+\sqrt{1+4|a|}}{2} .
\end{aligned}
$$

It follows that

$$
|u| \leq \frac{1+\sqrt{1+4|a|}}{2}
$$

and hence

$$
\left|\frac{R_{n+1}}{R_{n}}\right|<\sqrt{1-\frac{2 \gamma}{\left(1+\sqrt{1+4|a|)^{2}}\right.}} .
$$

Thus there exists $\varepsilon>0$ such that

$$
\left|\frac{R_{n+1}}{R_{n}}\right|<1-\varepsilon, \quad \text { for every } n,
$$

and therefore

$$
\lim _{n \rightarrow \infty} R_{n}=0 .
$$

If $\mathrm{I}(a)<0$, then $\alpha_{n+1}, \gamma>0$, and $\beta_{n+1}, \eta<0$, so that the above proof is still applicable. 
Case 1(b): a real, $a>-\frac{1}{4}$. In this case $\eta=0$, but otherwise the proof is the same as for Case 1(a), since even though $\alpha_{1}=0$ when $a<0$, $\alpha_{n}>0$ for all $n>1$.

Case 1(c): a real, $a<-\frac{1}{4}$. Here $\gamma=0, \eta>0, \alpha_{n}>0$ for $n>1$, $\beta_{n}>0$ for all $n$. Then

$$
\left|\frac{R_{n+1}}{R_{n}}\right|=\sqrt{1-\frac{4 \eta \beta_{n+1}}{\left|u_{n+1}+u\right|^{2}}} .
$$

We shall prove that there exists $\delta>0$ such that

$$
\beta_{n}>\delta \quad \text { for every } n \text {. }
$$

Let $\left|R_{n}\right|=k_{n}$. Notice that $k_{n}<1$ for every $n$, since

$$
R_{n}=\frac{u_{n}-\left(\frac{1}{2}+i \eta\right)}{u_{n}-\left(\frac{1}{2}-i \eta\right)},
$$

where $u_{n}$ is in the first quadrant and $\eta>0$.

The locus of points $z$ such that

$$
\left|\frac{z-u}{z-u^{*}}\right| \leq k_{n}
$$

is the circumference and interior of a circle (see, for example, [4], p. 192) with center

$$
\frac{1}{2}+i \frac{1+k_{n}^{2}}{1-k_{n}^{2}} \frac{\sqrt{4|a|-1}}{2}
$$

and radius

$$
\frac{k_{n} \sqrt{4|a|-1}}{1-k_{n}^{2}} .
$$

Since, by equation (1), $k_{n+1}<k_{n}$ for every $n$, each $u_{n}$ lies in or on the circle

$$
\left|\frac{z-u}{z-u^{*}}\right|=k_{1}
$$

Therefore, for every $n$,

$$
\begin{aligned}
\beta_{n} & \geq\left(\frac{1+k_{1}^{2}}{2\left(1-k_{1}^{2}\right)}-\frac{k_{1}}{1-k_{1}^{2}}\right) \sqrt{4|a|-1} \\
& =\frac{1-k_{1}}{2\left(1+k_{1}\right)} \sqrt{4|a|-1} .
\end{aligned}
$$


Hence there exists $\varepsilon>0$ such that

$$
\left|\frac{R_{n+1}}{R_{n}}\right|<1-\varepsilon, \quad \text { for every } n .
$$

Case 2: $a=-\frac{1}{4}$. The preceding proof is not applicable, since

$$
u=u^{*}=\frac{1}{2} \text {. }
$$

By equating real and imaginary parts in the equation

$$
u_{n+1}^{2}=u_{n}-\frac{1}{4}
$$

we obtain

$$
\begin{aligned}
& \text { (i) } \quad \alpha_{n+1}^{2}-\beta_{n+1}^{2}=\alpha_{n}-\frac{1}{4}, \\
& \text { (ii) } 2 \alpha_{n+1} \beta_{n+1}=\beta_{n} .
\end{aligned}
$$

First, $\alpha_{n}>\frac{1}{2}$ for all $n \geq 3$. The proof is by induction. If $\alpha_{n}>\frac{1}{2}$, then

$$
\alpha_{n+1}^{2}=\beta_{n+1}^{2}+\alpha_{n}-\frac{1}{4}>\frac{1}{4} .
$$

Also, $\alpha_{3}>\frac{1}{2}$ by direct computation.

Next, equation (ii) shows that

$$
\beta_{n+1}<\beta_{n}, \quad \text { for all } n \geq 3 .
$$

Because of our definition of the square root of a negative real number, it is clear that $\beta_{n}>0$ for every $n$. Therefore $\lim \beta_{n}$ exists and is greater than or equal to zero. Let $n \rightarrow \infty$

From the equation

$$
\beta=\lim _{n \rightarrow \infty} \beta_{n}
$$

we obtain

$$
u_{n+1}^{2}-\frac{1}{4}=u_{n}-\frac{1}{2},
$$

$$
u_{n \div 1}-\frac{1}{2}=\frac{u_{n}-\frac{1}{2}}{u_{n+1}+\frac{1}{2}} .
$$

For all $n \geq 3, \alpha_{n}>\frac{1}{2}$. Hence

and

$$
\left|u_{n+1}+\frac{1}{2}\right|>1
$$

$$
\left|u_{n+1}-\frac{1}{2}\right|<\left|u_{n}-\frac{1}{2}\right| \text {. }
$$

Then $\left\{\left|u_{n}-\frac{1}{2}\right|\right\}$ converges. Let

$$
\varrho=\lim _{n \rightarrow \infty}\left|u_{n}-\frac{1}{2}\right| .
$$

Now $\left\{u_{n}\right\}$ has at least one limit point because it is a bounded sequence. Every limit point of $\left\{u_{n}\right\}$ lies on the circle of radius $\varrho$ with center at $\frac{1}{2}$. 
But since $\lim _{n \rightarrow \infty} \beta_{n}=\beta$, the only possible limit points of $\left\{u_{n}\right\}$ are the points of intersection of the line $z=\beta i$ and the circle $\left|z-\frac{1}{2}\right|=\varrho$. Call these points $P$ and $Q$. The real coordinate of one of them, say of $P$, is less than or equal to $\frac{1}{2}$, while that of $Q$ is greater than or equal to $\frac{1}{2}$.

But since $\alpha_{n}<\frac{1}{2}$ for all $n \geq 3, Q$ is the only possible limit point of $\left\{u_{n}\right\}$. Therefore $\lim u_{n}$ exists. As we pointed out at the beginning of the proof of this theorem, if $\lim _{n \rightarrow \infty} u_{n}$ exists, it must be a root of

Therefore $\lim _{n \rightarrow \infty} u_{n}=\frac{1}{2}$.

$$
z^{2}-z+\frac{1}{4}=0 \text {. }
$$

\section{References}

1] Herschfeld, A.: On Infinite Radicals. - American Mathematical Monthly, Vol. 42 (1935) pp. 419-429.

[2] Myrberg, P. J.: Iteration von Quadratwurzeloperationen. - Annales Academiae Scientiarum Fennicae, Series AI, no. 259 (1958) pp. $1-16$.

[3] Schuske, G. and Thron, W. J.: Infinite Radicals in the Complex Plane. - Proceedings of the American Mathematical Society (Vol. 12 (1961) pp. 527-532).

[4] Thron, W. J.: Introduction to the Theory of Functions of a Complex Variable. New York, 1953.

University of Colorado

Boulder, Colorado, U.S.A. 